\title{
EI Valor Agregado Bruto, la corrupción y la desigualdad en Ecuador. Un análisis descriptivo y correlacional.
}

The Gross Value Added, corruption and inequality in Ecuador. A descriptive and correlational analysis.

Johanna Maricela Nievecela Lema. ${ }^{1}$, Daniele Covri Rivera. ${ }^{2} \&$ Yonimiler Castillo Ortega. $^{3}$

DOI: https://doi.org/10.33262/visionariodigital.v5i2.1634

\begin{abstract}
Introduction. This document analyzes the Gross Value Added (GVA) of the economic sectors of the Ecuadorian economy for the period 2000-2019, in order to determine the most influential sectors of the same; in addition to looking for a relationship between economic growth using the Gross Domestic Product (GDP) versus the Corruption Perception Index (CPI), which is the result of a phenomenon that is influenced by many other factors such as the economic one; later, a relationship will be developed between the inequality expressed by the Gini Coefficient and the CPI. The results obtained share agreement with research where it is evidenced that corruption acts as a brake on the increase in GDP, and with-it inequality. This research does not seek to give conclusions on how to improve the CPI and inequality, if it does not open the door to debate on the problem of corruption in Ecuador. Methodology. The research design was quantitative and qualitative, the first of a longitudinal non-experimental nature that allowed the analysis of the variables studied in the period of time. Collecting data from official

\footnotetext{
1 Universidad Católica de Cuenca, Maestría en Desarrollo Local, Cuenca, Ecuador, johannanievecela@ hotmail.com, https://orcid.org/0000-0002-4352-0853

2 Universidad Católica de Cuenca, Carrera de Economía, Cuenca Ecuador, daniele.covri@ucaue.edu.ec, https:orcid.org/0000-0002-2495-0399

3 Universidad Católica de Cuenca, Maestría en Desarrollo Local, ycastilloo@ucacue.edu.ec, https://orcid.org/0000-0002-7710-5199
} 
sources. Results. Among the results of the analysis of the Gross Value Added, the manufacturing sector stands out with a participation of 11.875 , followed by the commercial sector with $10.55 \%$ and the oil and mining sector with $10.42 \%$, in addition to the result of the correlation between GDP and Corruption Perception Index of 0.837 indicates a direct linear relationship; while for the correlation of the Corruption Perception Index and the Gini Coefficient there is an inverse linear relationship being the result of -0.731 . Conclusion. It was concluded that the manufacturing sector is the strongest in the Ecuadorian economy, however, the commerce and oil and mining sectors are not far behind the first; In addition, corruption and inequality have undermined the economic growth of Ecuador in the period analyzed.

Keywords: Gross Value Added; Corruption Perception Index, Economic Development; Gini coefficient

\section{Resumen}

Introducción. En el presente documento se analiza Valor Agregado Bruto (VAB) de los sectores económicos de la economía ecuatoriana para el periodo 2000-2019, para de esta manera determinar los sectores más influyentes de la misma; además de buscar una relación entre el crecimiento económico utilizando el Producto Interno Bruto (PIB) frente al Índice de Percepción de Corrupción (IPC) mismo que es el resultado de un fenómeno que se ve influenciado por muchos otros factores como lo es el económico; posteriormente se desarrollará una relación entre la desigualdad expresada por el Coeficiente de Gini con el IPC. Los resultados obtenidos comparten concordancia con investigaciones en donde se evidencia que la corrupción actúa como freno para el incremento del PIB, y con ello la desigualdad. Esta investigación no busca dar conclusiones de cómo mejorar el IPC y la desigualdad, si no da puerta al debate sobre el problema de corrupción existente en el Ecuador. Metodología. El diseño de investigación fue cuantitativa y cualitativa, el primero de carácter no experimental longitudinal que permitió el análisis de las variables estudiadas en el periodo de tiempo. Recopilando datos de fuentes oficiales. Resultados. Entre los resultados del análisis del Valor Agregado Bruto destaca el sector manufacturero con una participación del 11,875, seguido por el sector comercial con el $10,55 \%$ y el sector de petróleo y minas con el 10,42\%, además el resultado de la correlación entre PIB e índice de Percepción de Corrupción de 0.837 indica una relación lineal directa; en tanto para la correlación de índice de percepción de Corrupción y Coeficiente de Gini existe una relación lineal inversa la ser el resultado de -0.731 Conclusión. Se concluyó que el sector manufacturero es el más fuerte de economía ecuatoriana, sin embargo, los sectores de comercio y el petróleo y minas no se quedan muy atrás de la primera; además la corrupción y desigualdad han minado el crecimiento económico del Ecuador en el periodo analizado.

Palabras claves: Valor Agregado Bruto; índice de Percepción de Corrupción, Desarrollo Económico; Coeficiente de Gini. 


\section{Introducción.}

La economía de las naciones ha sido cambiante gracias a las políticas fiscales empleadas en base a los distintos escenarios particulares que cada país posee; además de las externalidades como: crisis mundiales, caída del precio del petróleo, entre otros. El desarrollo económico es de vital importancia para todas las naciones del mundo, pero en especial se evidencia su importancia en las economías subdesarrolladas como es el caso de Latinoamérica que se ha caracterizado por ser primario exportador. Además, las medidas implantadas obedecen a factores externos, siendo la venta de commodities el rubro más alto en sus balanzas comerciales; al igual que, la economía ecuatoriana ya que se ha caracterizado por un modelo de desarrollo hacia afuera.

El desarrollo económico, ha sido un campo de amplio debate y preocupación durante muchas décadas por parte de las naciones; en donde, han surgido diversos pensadores, escuelas y teorías para ayudar a cumplir el reto del desarrollo. Cabe destacar que el tan ansiado desarrollo económico, no todas las naciones lo han logrado. El desarrollo de las naciones se ha concebido a raíz de la industrialización, es decir, añadiendo un valor agregado a sus productos en estado natural. El desarrollo y el crecimiento económico buscan el cambio dentro de la estructura económica, en sí el objetivo del desarrollo económico es lograr la prosperidad dentro de un entorno específico.

Según Hofman, (1994) Ecuador a partir de la primera y segunda década del siglo XX tuvo un crecimiento económico lento debido a la Primera Guerra Mundial. A pesar de ello tuvo el crecimiento más rápido de la región, mismo que fue impulsado por el Cacao. Entre la tercera y quinta década del siglo XX las exportaciones se redujeron a más de la mitad, esto representó una disminución en las importaciones, sumándose una inestabilidad política; logrando estabilidad política a finales de los años 40. Entre la quinta y séptima década del siglo XX la economía ecuatoriana se vio beneficiada por el incremento de la producción de banano. Dentro de la década de los años 50 el modelo agroexportador fue remplazado por el modelo de industrialización sustitutiva de importaciones con el fin de generar un proceso de desarrollo económico autónomo del sector externo. A mediados de los 60 se iniciaron políticas dirigidas a la acumulación interna favoreciendo así el sector industrial, es así como se introduce una reforma agraria basada en los postulados de alianza para el progreso dominantes en América Latina. En los años 70 empezó el boom petrolero, mismo que dinamizó la economía ecuatoriana. En la década de los 80 se originó a falta de diversificación de importaciones, desencadenándose un estancamiento de las exportaciones, y en la postergación de incrementar el ingreso no petrolero y la incapacidad de atraer capital extranjero (Montalvo, 2017).

Para los años 90, el promedio de crecimiento del producto interno bruto (PIB) ecuatoriano fue del 1,8\%, siendo su punto más alto en 1991 con 5,2\%, y su punto más bajo en 1999 con un decrecimiento del $6.3 \%$, debido a la caída del precio del petróleo que se ubico en 9 dólares el barril. Por otro lado, el fenómeno del niño azotaba el litoral ecuatoriano, el feriado bancario, la escalada del dólar y una elevada inflación; misma que se ubico en el $59,9 \%$, para mitigar los efectos de la desvalorización de la moneda propia (Sucre). Se 
estableció adoptar el dólar como moneda y para el año 2000 la economía ecuatoriana se acogió a la dolarización. En la primera década el crecimiento promedio se ubico en el $4.4 \%$, la economía ecuatoriana creció debido al incremento del valor agregado petrolero (VAP).

Para el periodo posterior, se evidencia un mayor crecimiento de la manufactura que se encuentra dentro del VAB no petrolero; en cierta medida, se intentó alinear al pensamiento Cepalino de desarrollo hacia adentro. Los intentos del Estado para lograr un proceso de desarrollo hasta el momento han sido infructíferos, a pesar de que se han logrado avances no se ha cambiado el modelo de desarrollo acogido en el siglo XX. No obstante, en términos de exportación, según el BCE, el rubro predominante ha sido el sector primario. Desde el 2015 a 2019 la participación petrolera dentro de las exportaciones ha sido del $37 \%$; las exportaciones no petroleras tradicionales aportaron el $37 \%$; y, finalmente las exportaciones no petroleras no tradicionales con el $27 \%$.

Por lo antes expuesto se evidencia que desde el siglo XX, el Ecuador se ha enfocado en la venta de materia prima, empezando por productos agrícolas como: banano y cacao mismos que en su momento fueron la mayor fuente de ingreso del país. Posteriormente, ese lugar lo tomó el petróleo, que es la fuente de mayor ingreso para el país hasta el momento. Desde la constitución como república, no se ha podido cambiar la estructura económica, a pesar del intento de cambio de matriz productiva que se vivió en los últimos años. Es por esta razón que es importante analizar la estructura económica ecuatoriana para establecer las determinantes que han influenciado en el desarrollo de la economía ecuatoriana durante las dos primeras décadas del siglo XXI; y así explicar la razón de por que se ha mantenido un modelo primario exportador, lo cual se aborda en la presente investigación.

\section{Marco Teórico.}

El desarrollo económico para (Marjanović, 2016) implica crear nuevas características de calidad, lo cuál requiere cambios estructurales. Esto conduce al problema del desarrollo entre las naciones motivando la separación entre las economías desarrollas y subdesarrolladas, ya que las primeras tienen mayor ventaja sobre las segundas debido a que poseen mayores recursos económicos y tecnológicos que potencian su desarrollo.

En tanto para Valdez (2016) y Astroulakis (2013) el desarrollo requiere de un cambio cualitativo, el fin del desarrollo es lograr una sociedad próspera. Además, se plantea que la medición del crecimiento económico de un país se basa en el $\mathrm{PIB}^{4}$. Este indicador nos da una visión general de la situación económica que se vive dentro de un territorio; más no nos describe la situación social por la que éste atraviesa. Sin embargo, el desarrollo económico tiende a no ser igualitario dentro del mismo territorio, ya que se generan polos de desarrollo, mismos que hacen destacar a una parte de la región más que a otra. Lo

\footnotetext{
${ }^{4}$ Producto Interno Bruto
} 
conveniente es generar encadenamientos productivos, esto es que las regiones aledañas sean las proveedoras de materiales al polo de desarrollo.

\section{Tendencias Teóricas sobre Desarrollo Económico.}

Los primeros esbozos sobre las teorías del desarrollo empezaron con los griegos, siendo ellos quienes sentaron las bases teóricas de la economía. Martínez \& Crespo (2011) consideran que Aristóteles fue el pionero en introducir conceptos básicos de economía. (Vergara \& Ortiz, 2016) consideran que a partir del siglo XVII empieza la preocupación sobre el desarrollo y se reconoce a los mercantilistas como pioneros del desarrollo, seguidos por los fisiócratas quienes introdujeron conceptos como producción y consumo. Con esto se evidencia la preocupación por el desarrollo desde los inicios de la sociedad, ya que siempre se ha buscado vivir en mejores condiciones como grupo humano.

Dentro del debate clásico según señalan Astroulakis (2019) y Restrepo (2015) se considera importante resaltar el aporte de Adam Smith en su obra "Investigación de la naturaleza y riqueza de las naciones“, en la cual expone conceptos como: mano invisible y división del trabajo, lo cual fundamenta la idea de que cada territorio debe dedicarse a producir un solo bien o servicio, lo cual implica especializarse en una sola actividad. David Ricardo por su parte postuló la teoría de los factores de producción: tierra, capital y trabajo.

La teoría de la modernización buscaba cambiar la estructura económica dejando de lado la comercialización de materia prima, dando paso a la industrialización. Franco \& Ramirez, (2005) señalan que para Harrod y Domar uno de los problemas que se podría presentar es la escasez de la mano de obra, más la escasez de inversión. Para Solow era importante el papel que tomaba el capital, además del crecimiento demográfico y el adelanto tecnológico. Kutztents señala que el desarrollo económico se mide mediante el PIB. Según Lewis el crecimiento económico debe basarse en la igualdad. Finalmente Ornelas (2012) señala que Rostow considera que para lograr el despegue inicial, todas las naciones deben pasar por un proceso de transición, para de esta manera lograr el desarrollo.

La teoría Cepalina obtuvo un papel importante en la historia del desarrollo económico a partir de la creación de la Comisión Económica de las Naciones Unidas para América Latina (CEPAL). Según señalan CEPAL (2016); Frank (2005) y Beteta \& Moreno-Brid (2012) durante la década de los cincuenta se planteó a la industrialización como la impulsora del desarrollo. En los años sesenta se enfocó su planteamiento a la reducción de desigualdades. En los años setenta se abre el debate sobre los estilos de desarrollo. La década de los años 80 es considerada la década perdida ya que no se hace referencia la desarrollo si no más bien a temas macroeconómicos; y para los 90 el mensaje fue transformación productiva con equidad. Un tema fundamental para las economías subdesarrolladas es el deterioro de términos de intercambio ya que los países de la "periferia“ venden materias primas al "centro" a bajo costo; y el "centro" vende productos terminados a un alto costo; es ahí donde se produce una disparidad; por lo que se reafirma a la tecnología como un factor importante para lograr el desarrollo. 
Bielschowsky (2006) revisa los aportes de Celso Furtado a la teoría cepalina en donde se señala que puede existir resistencia por parte de ciertos grupos económicos que comercializan productos del sector primario, debido a que perderían mercado e ingresos y se establece que el subdesarrollo en América Latina está conformado por 2 factores: el bajo ingreso y la notable diferencia de clases sociales. Para Petit (2014) la teoría de la integración de países puede ser fructífera, bajo la condición de paridad de crecimiento, es decir todos los países miembros se apoyan implementando libre comercio y ausencia de aranceles a la importación o exportación; lo cual dinamizaría la economía de los países miembros de la unión, para de esta manera sustituir las importaciones de los países que no son miembros.

La teoría de la globalización para Reyes (2001) ha sido un factor importante para el desarrollo de las naciones, esto se explica ya que a través de la implementación de la tecnología ha sido posible tecnificar procesos, del mismo modo ha sido posible reducir costos y brindar productos de mejor calidad. La tecnología ha producido un cambio económico y social tanto en los países desarrollados como los que se encuentran en vías de desarrollo.

Para Iturralde (2019) y Sen Amartya (2009) el Desarrollo Humano Sostenible para el Programa de Naciones Unidas para el Desarrollo (PNUD) se basa en combinar tres factores que son: económico, social y el respeto a la naturaleza. Esto implica un enfoque pionero, ya que incorpora a la naturaleza como un determinante para el desarrollo. Es decir, la economía debería propender a que el crecimiento económico vaya de la mano del respeto del medio ambiente, buscando un cambio hacia la economía verde. El PNUD busca igualdad dentro del desarrollo derribando barreras de genero y anteponiendo al ser humano por sobre el dinero. Este planteamiento propende a brindar igualdad a los seres humanos derribando barreras obsoletas generadas por la sociedad; para de esta manera forjar una nueva sociedad con conciencia social y ecológica.

\section{Aspectos teóricos sobre el valor agregado bruto, el índice de corrupción y el coeficiente de Gini.}

El Valor Agregado Bruto (VAB) para (Guerra \& González, 2015) es la expresión del crecimiento económico, ya que este es un indicador equivalente al PIB y es el resultado de una actividad de producción misma que tiene en cuenta un conjunto de bienes y servicios, descontando impuestos indirectos y consumo intermedio.

Vélez (2018) señala que la división económica por sectores tuvo lugar en el siglo XX con la formulación de la Ley de Petty-Clark en dónde se señalan tres sectores: primario, secundario y terciario. Rodero (2012) explica que el sector primario está relacionado con la obtención de los productos en estado puro de la tierra o mar. Dentro de esta clasificación encontramos a la agricultura, ganadería, silvicultura, caza y pesca. Es decir, estos productos no han sufrido un proceso de transformación y se expenden en estado puro sin la incorporación de un valor agregado mediante procesos de industrialización o tratamiento para la exportación del bien en estado puro. Este modelo se caracteriza por 
su incapacidad de generar un valor agregado a sus bienes o servicios; en los países subdesarrollados este es el modelo de prevalencia.

El sector secundario o industrial para Banco Mundial (2016) y Roark, Urrutia, Jaureguiberry, Paravié \& Ottogalli. (2013) comprenden actividades de transformación de los recursos naturales y se producen bienes intermedios, finales y bienes de capital. Es decir, contempla la industrialización de la materia prima para de esta manera convertirlas en productos intermedios o terminados.

El sector terciario o de servicios según Schmidtke et al. (2014) abarca servicios hoteleros, restaurants, transporte, administración pública, servicios profesionales y actividades inmobiliarias. La ONU (2005) y el Instituto Nacional de Estadísticas y Censos (INEC) del Ecuador contextualizan la división económica por sectores primario, secundario y terciario en donde cada uno presenta una desagregación, donde cada actividad económica suma a un sector determinado.

Transparencia Internacional a partir del año 1995 publica datos sobre el Índice de Percepción de Corrupción (IPC); este índice se mide a partir del criterio de personas de negocios, sobre la percepción de la corrupción en el país en donde se encuentran sus empresas, además se toma en cuenta el criterio de analistas económicos; los países con alta calificación son los percibidos como más corruptos y por lo general poseen un bajo desarrollo humano, lo cual indica una posible relación entre corrupción y desarrollo.(Campos \& Pradhan, 2009)

Para Bautista (2012) la corrupción es un fenómeno reconocido a nivel mundial, ya que conlleva efectos adversos sobre la estructura social, política y económica de las naciones que la adolecen. Este reduce la eficiencia de la institucionalidad del Estado, además de los problemas sociales que acarrean consigo procesos políticos y valores dañados, acompañado de la pobreza en ascenso. Para (Palacios, 2014) dentro de su estudio se determina que la corrupción es un obstáculo para el crecimiento económico, además del deterioro del bienestar social por el aumento de la desigualdad, ya que existe una acumulación de riqueza en determinados grupos. En tanto Hernández \& Herrera (2018) de un análisis para 75 países determinan la existencia indisputable de la retroalimentación simultanea entre el crecimiento económico y el IPC existentes en las instituciones públicas, es decir a menor crecimiento económico se evidencia un mayor IPC.

Para el caso Colombiano Palestina (2018) concluye en su investigación que la economía presenta un estancamiento por tanto el IPC también, pero en relación a periodos anteriores se evidencia cierta mejoría en cuanto al IPC, por lo que se nos demuestra que a mayor corrupción existente se deteriora el crecimiento económico del país. En el caso Mexicano López \& Sánchez (2013) concluyen que la corrupción tiene un efecto negativo directo sobre el crecimiento económico y está directamente relacionado con las instituciones públicas.

En América Latina los sobornos se destinan para el sistema judicial, policía, sistema educativo, servicio de registro o permisos y finaliza con el sector público. Además 
Wielandt \& Artigas (2007) concluye que el IPC tiene una fuerte correlación con la pobreza, es decir a la mayor pobreza mayor corrupción en su estudio realizado para América Latina y el Caribe. Ortiz (2012) señala que la corrupción afecta negativamente a los servicios sociales prestados por el estado como lo son la educación y la salud para el caso colombiano.

El Índice de Percepción de Corrupción es un indicador que mide las expectativas que tiene la población de un determinado país con respecto al nivel de transparencia en las instituciones. Toma un valor que va desde 0 a 100, siendo: ausencia de transparencia (completa corrupción) cuando 1 IPC toma el valor de 0; completa transparencia (sin corrupción) cuando el IPC toma el valor de 100. (Transparecia Internacional, 2014)

(Núñez, 2006) Señala que el Coeficiente de Gini es un indicador que sirve para medir la desigualdad a partir de la diferencia media de las rentas de la distribución, se utiliza para medir las diferencias en la distribución de la renta; y viene dado entre los valores de 0 y 1; a medida que aumenta la desigualdad el coeficiente de gini se acerca al valor de 1; y mientras más cerca de 0 la desigualdad decrece. (Medina, 2001).

La corrupción y la desigualdad son factores que afectan al desarrollo económico como se hace referencia en los anteriores casos. De manera especial América Latina vive problemas enraizados de corrupción presentes en las instituciones manejadas por el Estado, ya que los procesos no se llevan con transparencia y en ocasiones se incurre en perjuicio económico para el estado central y a la vez esto genera más desigualdad entre los habitantes de un país ya que se hacen aun más visibles las brechas: sociales, económicas y de género.

\section{Metodologia.}

La presente investigación es mixta, ya que se utilizaron los enfoques cuantitativo y cualitativo. El primero de carácter no experimental longitudinal permitió los análisis estadísticos correlacionales de las variables estudiadas en el periodo 2000 - 2019 y un análisis descriptivo del VAB del Ecuador encontrándose los sectores económicos que más aportaron a la economía nacional en el período de análisis. La metodología empleada fue la fórmula de variación anual, presentandose así un análisis de los componentes del VAB mediante un análisis descriptivo, en donde se hace enfasis en los sectores más imporatantes que dinamizaron la economía en este periodo.

La base de datos empleada para este análisis se construyó en base a información del Banco Central del Ecuador (BCE), a partir del año 2000 hasta el año 2019 tomando en cuenta cada sector económico que forman el VAB. La tecnica descriptiva del análisis de los datos se enfoca en resaltar los sectores con mayor influencia dentro de la económia ecuatoriana, al igual que los sectores que menor influencia poseen.

Para calcular la relación lineal existente entre las variables se utilizó el Índice de Percepción de Corrupción (IPC); y el Coeiciente de Gini y el Producto Interno Bruto real (PIB real); se utilizó el coeficiente de correlación lineal de Pearson, cuya fórmula es: 


$$
r_{x y}=\frac{\sum Z_{x} Z_{y}}{N}
$$

Restrepo \& González (2007) indican indican que el coeficiente de correlación lineal de Pearson es igual a la sumatoria de los productos de las puntuaciones estandarizadas de cada variable dividido para el tamaño muestral. El valor del coeficiente toma valores entre -1 y 1; de tal manera que, cuando el coeficiente es negativo indica que entre las variables analizadas existe una relación inversa; cuando el coeficiente es positivo indica la existencia de una relación directa y si el coeficiente tomar valor cero, es un indicativo de que no existe ninguna relación lineal entre las variables.

Cabe señalar que el coeficiente de correlación de Pearson es únicamente una medida del nivel de relación lineal existente entre 2 variables, por lo que sus resultados no se pueden considerar un indicativo de causa y efecto. Las variables utilizadas para el análisis de correlación son: el Índice de Percepción de Corrupción y el PIB real, cuyos datos han sido recopilados anualmente, a partir del año 2000 y hasta el 2019. Estos datos se han recopilado de fuentes fidedignas como los son el Banco Central del Ecuador y data del Banco Mundial; posteriormente se realizó una correlación entre el Coeficiente de Gini, y el índice de percepción de corrupción.

La correlación refleja el grado de relación que estas variables poseen sobre el desarrollo económico del país; se utilizó el programa de código abierto R Studio para el calculo de la correlación, además de la incorporación de histogramas para evidenciar graficamente la evolución de estas variables a lo largo del periodo de estudio.

\section{Resultados.}

El PIB en el año 2011 presenta una tasa de crecimiento de 7,29\% en relación con el año anterior; para 2004 la tasa de crecimiento se ubicó en el 7,59\%; mientras que para 2016 se registró un decrecimiento de la tasa del 1,24\%; la tasa promedio de crecimiento del PIB para el Ecuador durante el periodo 2000 - 2019 es del 3,31\%.

El promedio de participación durante el periodo 2000- 2019 es el siguiente:

La manufactura representa el 11,87\% del PIB; el comercio el 10,55\%; petróleo y minas 10,42\%; construcción 8,39\%; enseñanza y servicios sociales y de salud 8,21\%; agricultura, ganadería, caza y silvicultura 7,92\%; otros servicios 7,51\%; transporte 6,75\%; actividades profesionales, técnicas y administrativas 6,11\%; Administración pública, defensa 5,94\%; otros elementos del PIB 4,17\%; correo y comunicaciones 2,73\%; actividades de servicios financieros 2,69\%; suministro de electricidad y agua 1,82\%; alojamiento y servicios de comida $1,72 \%$; refinación del petróleo $1,67 \%$, pesca excepto camarón $0,67 \%$; acuicultura y pesca de camarón $0,57 \%$ y Servicio doméstico 0,30\%.

A continuación, se detallará el comportamiento de los 5 sectores mas importantes para la economía ecuatoriana, tenemos así: 
El sector manufacturero presenta en el año 2008 del 8,40\%; para el año 2005 el crecimiento registrado fue del 6\%; en tanto para el 2013 fue del $5.80 \%$, presentado un decrecimiento importante para el año 2016 del 2,67\%; registrando una tasa de crecimiento promedio del 3,16\% durante el periodo de estudio. El valor más alto por concepto de manufactura se registra para el año 2019 con 8.489 .579 dólares, en tanto que el valor más pequeño se encuentra en el año 2000 con 4.581 .123 dólares.

En cuanto al sector comercial la mayor tasa de crecimiento registrada se ubica para el año 2008 con un crecimiento del 9,72\%; para el año 2013 la tasa de crecimiento registrada fue del 6,35\%; para 2005 la tasa de crecimiento fue del 5,71\%; por otro lado, la tasa de decrecimiento más alta que se registró fue para el año 2016 con un decrecimiento del $4,13 \%$ con relación al año anterior; la tasa promedio de crecimiento anual para el periodo analizado es del 2,77\%. Para el año 2018 el ingreso por concepto de comercio fue de 7.372.446 dólares; y para 2019 fue de 7.308 .895 dólares evidenciándose entre estos 2 años un decrecientito del $0,87 \%$.

El sector petróleo y minas presentó la mayor tasa de crecimiento registrada es del año 2004 con el 29,44\%; para el año 2003 la tasa de crecimiento se ubicó en el 9,72\%; y para el año 2014 el crecimiento registrado fue de 6,18\%; finalmente se puede destacar un decrecimiento para el año 2007 con el 8,16\%; la tasa promedio de crecimiento anual es del 2,33\%. En el año 2014 se produjo el mayor ingreso de este sector con 6.889.067 dólares; el menor ingreso se obtuvo en el año 2002 con 3.815.780 dólares.

El sector de la construcción presentó la mayor tasa de crecimiento para el año 2001 con el18.90\% con relación al año anterior; para el año 2002 la tasa de crecimiento se ubicó en el 17,33\%; en tanto para 2011 con el 14,93\%; registrándose un decrecimiento para el año 2016 del 6,12\%; la tasa promedio anual de la construcción para el periodo analizado es del 4,65\%. Para el año 2014 se evidencia el mayor ingreso por concepto de construcción siendo de 6.893 .456 dólares; seguido muy de cerca por el valor del año 2015 con 6.838.747 dólares; en tanto que el año 2000 fue el que registró el valor más bajo con 2.253.623 dólares.

Enseñanza y Servicios sociales y de salud: La mayor tasa de crecimiento registrada en el periodo de estudio se aprecia en el año 2009 con el 7,20\%; para el año 2010 fue de 6,71\% en relación con el año anterior; para 2012 se ubicó en el 6,37\%; además se registró un decrecimiento para el año 2002 del 1,01\%; la tasa promedio anual de crecimiento se ubicó en el 3,56\%. En el año 2018 este sector aportó a la economía con 6.449.486 dólares; en 2019 fue de 6.416 .318 dólares siendo notorio un decremento entre estos 2 años, registrando una tasa de decrecimiento del $0.52 \%$.

En tanto los sectores con menor incidencia sobre el PIB son:

Acuicultura y pesca de camarón: La mayor tasa de crecimiento registrada fue en el año 2005 con un crecimiento del 30,93\%; para 2014 se registró un crecimiento del 28,59\%; en el año 2003 se evidenció un crecimiento del 19,72\%; para el año 2011 el crecimiento fue de $17,86 \%$; por otra parte, la menor tasa de crecimiento registrado fue en el año 2002 
con el $0,99 \%$; la tasa promedio de crecimiento anual para el sector es del $12.10 \%$. Para el año 2019 el sector generó 913.487 dólares; para 2018 fue de 814.729 dólares; en tanto para el año 2000 fue de 72.153 dólares.

Servicio doméstico: para el año 2009 se evidenció la mayor tasa de crecimiento con el 14,07\%; para el año 2016 existió un crecimiento del 7,89\% con respecto al año anterior; en el año 2017 la tasa de crecimiento se ubicó en el 7,36\%; presentando un decrecimiento para el año 2005 del 4,71\%; la tasa anual promedio de crecimiento se ubicó en el 2,55\% para el periodo analizado. El servicio doméstico aportó a la economía nacional 213.347 dólares, en 2018 aportó con 211.938 dólares, produciendo un decrecimiento entre estos años del $0,66 \%$.

\begin{tabular}{|c|c|c|c|c|c|c|c|c|c|c|c|c|c|c|c|c|c|c|c|c|}
\hline \multicolumn{21}{|c|}{ Variación porcentual de los sectores del Valor Agregado Bruto para el periodo 2000 - 2019} \\
\hline Período & 2000 & 2001 & 2002 & 2003 & 2004 & 2005 & 2006 & 2007 & 2008 & 2009 & 2010 & 2011 & 2012 & 2013 & 2014 & 2015 & 2016 & 2017 & 2018 & 2019 \\
\hline $\begin{array}{l}\text { Agricultura, } \\
\text { ganadería, } \\
\text { caza y } \\
\text { silvicultura }\end{array}$ & 0,08 & 0,09 & 0,08 & 0,09 & 0,08 & 0,08 & 0,08 & 0,08 & 0,08 & 0,08 & 0,08 & 0,08 & 0,07 & 0,07 & 0,08 & 0,08 & 0,08 & 0,08 & 0,08 & 0,08 \\
\hline $\begin{array}{l}\text { Acuicultura y } \\
\text { pesca de } \\
\text { camarón }\end{array}$ & 0,00 & 0,00 & 0,00 & 0,00 & 0,00 & 0,00 & 0,00 & 0,00 & 0,00 & 0,00 & 0,00 & 0,01 & 0,01 & 0,01 & 0,01 & 0,01 & 0,01 & 0,01 & 0,01 & 0,01 \\
\hline $\begin{array}{l}\text { Pesca (excepto } \\
\text { camarón) }\end{array}$ & 0,01 & 0,01 & 0,01 & 0,01 & 0,01 & 0,01 & 0,01 & 0,01 & 0,01 & 0,01 & 0,01 & 0,01 & 0,01 & 0,01 & 0,01 & 0,01 & 0,01 & 0,01 & 0,01 & 0,01 \\
\hline $\begin{array}{l}\text { Petróleo y } \\
\text { minas }\end{array}$ & 0,10 & 0,10 & 0,09 & 0,10 & 0,13 & 0,13 & 0,13 & 0,12 & 0,11 & 0,11 & 0,11 & 0,10 & 0,10 & 0,10 & 0,10 & 0,10 & 0,10 & 0,09 & 0,09 & 0,09 \\
\hline $\begin{array}{l}\text { Refinación de } \\
\text { Petróleo }\end{array}$ & 0,03 & 0,03 & 0,03 & 0,02 & 0,02 & 0,02 & 0,02 & 0,02 & 0,02 & 0,02 & 0,02 & 0,02 & 0,02 & 0,01 & 0,01 & 0,01 & 0,01 & 0,01 & 0,01 & 0,01 \\
\hline $\begin{array}{l}\text { Manufactura } \\
\text { (excepto } \\
\text { refinación de } \\
\text { petróleo) }\end{array}$ & 0,12 & 0,12 & 0,12 & 0,12 & 0,12 & 0,12 & 0,12 & 0,12 & 0,12 & 0,12 & 0,12 & 0,12 & 0,12 & 0,12 & 0,12 & 0,12 & 0,12 & 0,12 & 0,12 & 0,12 \\
\hline $\begin{array}{l}\text { Suministro de } \\
\text { electricidad y } \\
\text { agua }\end{array}$ & 0,01 & 0,01 & 0,01 & 0,01 & 0,01 & 0,01 & 0,01 & 0,01 & 0,01 & 0,01 & 0,02 & 0,02 & 0,02 & 0,02 & 0,02 & 0,03 & 0,03 & 0,03 & 0,03 & 0,03 \\
\hline Construcción & 0,06 & 0,07 & 0,08 & 0,08 & 0,08 & 0,08 & 0,08 & 0,08 & 0,08 & 0,08 & 0,08 & 0,09 & 0,10 & 0,10 & 0,10 & 0,10 & 0,09 & 0,09 & 0,09 & 0,08 \\
\hline Comercio & 0,11 & 0,11 & 0,11 & 0,11 & 0,11 & 0,11 & 0,11 & 0,11 & 0,11 & 0,10 & 0,10 & 0,10 & 0,10 & 0,10 & 0,10 & 0,10 & 0,10 & 0,10 & 0,10 & 0,10 \\
\hline $\begin{array}{l}\text { Alojamiento y } \\
\text { servicios de } \\
\text { comida }\end{array}$ & 0,02 & 0,02 & 0,02 & 0,02 & 0,02 & 0,02 & 0,02 & 0,02 & 0,02 & 0,02 & 0,02 & 0,02 & 0,02 & 0,02 & 0,02 & 0,02 & 0,02 & 0,02 & 0,02 & 0,02 \\
\hline $\begin{array}{l}\text { Transporte } \\
\text { Correo y } \\
\text { Comunica- } \\
\text { ciones }\end{array}$ & 0,07 & 0,07 & 0,07 & 0,07 & 0,06 & 0,06 & 0,06 & 0,06 & 0,06 & 0,07 & 0,07 & 0,06 & 0,06 & 0,07 & 0,07 & 0,07 & 0,07 & 0,07 & 0,07 & 0,07 \\
\hline $\begin{array}{l}\text { Actividades de } \\
\text { servicios } \\
\text { financieros }\end{array}$ & 0,02 & 0,02 & 0,02 & 0,02 & 0,02 & 0,02 & 0,03 & 0,03 & 0,03 & 0,03 & 0,03 & 0,03 & 0,03 & 0,03 & 0,03 & 0,03 & 0,03 & 0,03 & 0,03 & 0,04 \\
\hline $\begin{array}{l}\text { Actividades } \\
\text { profesionales, } \\
\text { técnicas y } \\
\text { administrativas }\end{array}$ & 0,05 & 0,06 & 0,06 & 0,06 & 0,06 & 0,06 & 0,06 & 0,06 & 0,06 & 0,06 & 0,06 & 0,06 & 0,06 & 0,06 & 0,06 & 0,06 & 0,06 & 0,06 & 0,06 & 0,06 \\
\hline $\begin{array}{l}\text { Enseñanza y } \\
\text { Servicios } \\
\text { sociales y de } \\
\text { salud }\end{array}$ & 0,08 & 0,08 & 0,08 & 0,08 & 0,08 & 0,08 & 0,08 & 0,08 & 0,08 & 0,08 & 0,09 & 0,08 & 0,08 & 0,08 & 0,08 & 0,08 & 0,09 & 0,09 & 0,09 & 0,09 \\
\hline $\begin{array}{l}\text { Administración } \\
\text { pública, } \\
\text { defensa; } \\
\text { planes de } \\
\text { seguridad } \\
\text { social } \\
\text { obligatoria }\end{array}$ & 0,06 & 0,06 & 0,06 & 0,06 & 0,05 & 0,05 & 0,05 & 0,05 & 0,05 & 0,06 & 0,06 & 0,06 & 0,06 & 0,06 & 0,06 & 0,06 & 0,07 & 0,07 & 0,06 & 0,06 \\
\hline $\begin{array}{l}\text { Servicio } \\
\text { doméstico }\end{array}$ & 0,00 & 0,00 & 0,00 & 0,00 & 0,00 & 0,00 & 0,00 & 0,00 & 0,00 & 0,00 & 0,00 & 0,00 & 0,00 & 0,00 & 0,00 & 0,00 & 0,00 & 0,00 & 0,00 & 0,00 \\
\hline
\end{tabular}




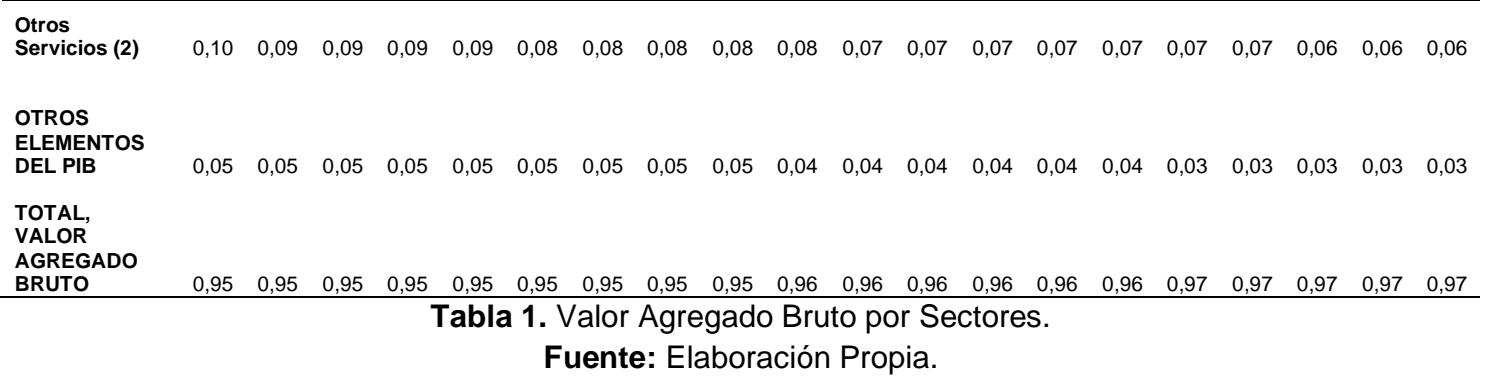

Correlación entre el Producto interno Bruto y el Índice de Percepción de Corrupción.

Al graficar la serie del Índice de Percepción de Corrupción, se observa que al inicio del período se mantiene constante, sin embargo, es a partir de 2008 que se aprecia un comportamiento claramente creciente, lo cual significa que en el Ecuador ha aumentado el nivel de transparencia, es decir, ha disminuido la corrupción.

Índice de Percepción de Corrupción

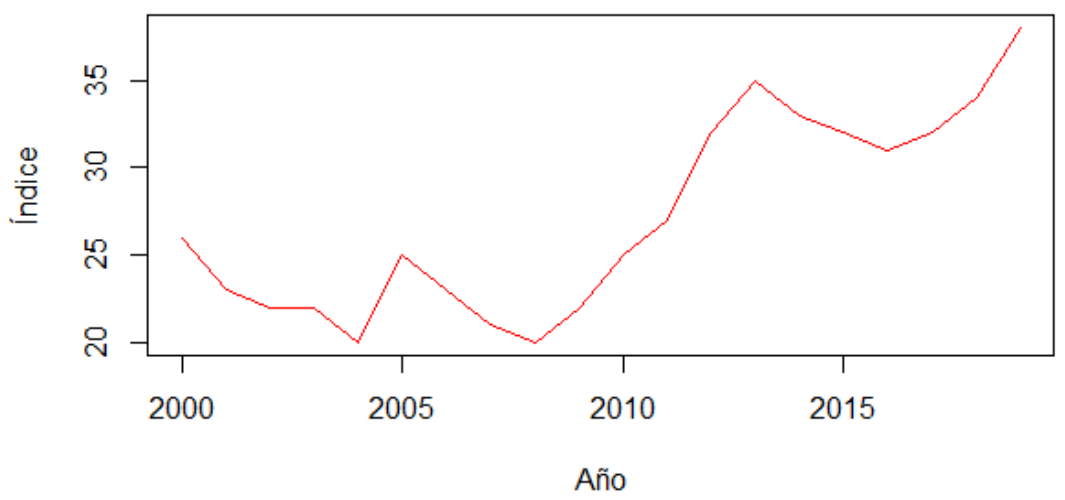

Figura 1. Indice de Percepción de Corrupción.

Fuente: Elaboración Propia.

Al observar la serie del PIB real, se puede ver un claro comportamiento también alcista en todo el período de análisis, siendo su punto más alto en el año 2019.

PIB REAL

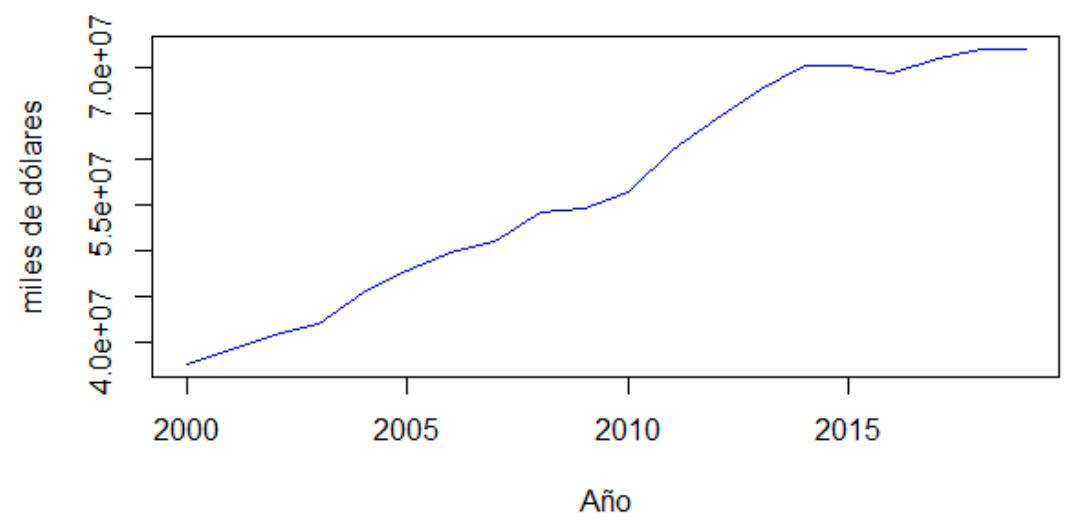

Figura 2. PIB real

Fuente: Elaboración Propia. 
Al graficar un diagrama de dispersión entre las dos variables, y trazar una línea de tendencia, se puede apreciar que existe una aparente relación lineal directa entre las variables, lo que a priori nos haría pensar que el coeficiente de correlación de Pearson debería ser positivo.

\section{Diagrama de Dispersión}

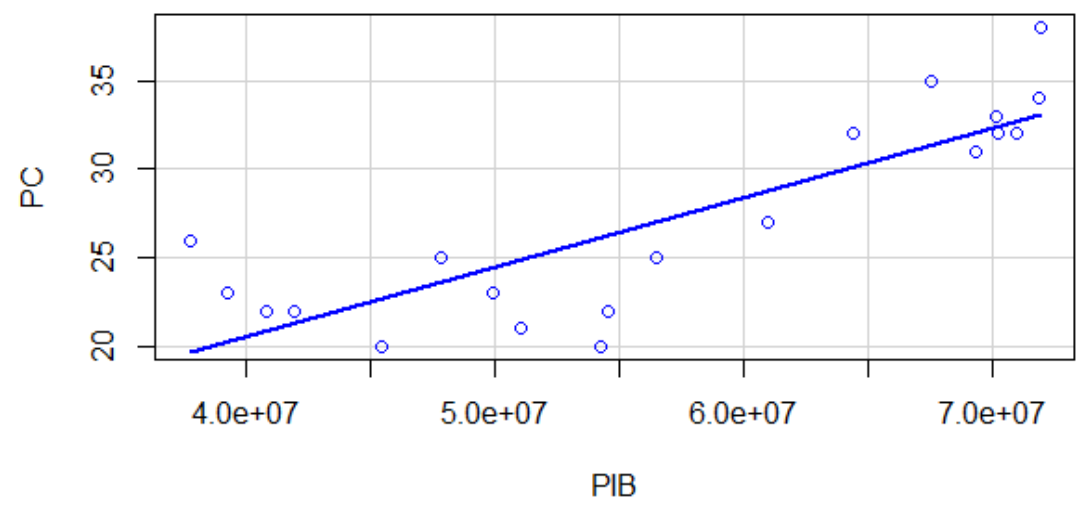

Figura 3. Diagrama de dispersión Fuente: Elaboración Propia.

Finalmente se calcula el coeficiente de correlación lineal de Pearson, lo cual arroja el siguiente resultado:

$$
r_{x y}=0.8370146
$$

Esto indica que existe una relación lineal directa entre las variables: Índice de Percepción de Corrupción y el PIB real, y dicha relación es bastante alta. Esto significa que, cuando una variable aumenta la otra también lo hace, es decir, las variables se mueven en el mismo sentido. Esto nos comprueba que a mayor sea la percepción de la corrupción el creciemiento económico se verá afectado negativamente.

Cuando el Índice de Percepción de Corrupción aumenta, es decir aumenta la transparencia de las instituciones (disminuye la corrupción), el PIB real también aumenta; sin embargo, este resultado no implica existencia de causalidad, es decir, esto no quiere decir que los cambios en el Índice de Percepción de Corrupción tengan un efecto sobre el PIB real, o viceversa, simplemente muestra que, para el período de análisis, existe una relación matemática directa entre las dos variables.

\section{Correlación entre índice de Percepción de Corrupción y Coeficiente de Gini.}

Al observar la serie del Coeficiente de GINI se puede ver un claro comportamiento hacia la baja en todo el período de análisis siendo su punto más bajo en el año 2018. 


\section{Coeficiente de GINI}

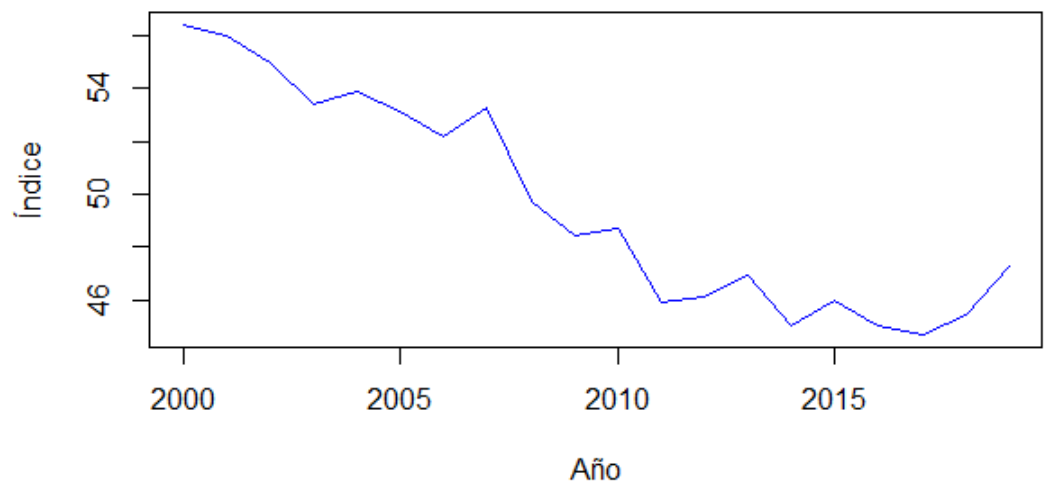

Figura 4. Coeficiente de Gini.

Fuente: Elaboración Propia.

Al graficar un diagrama de dispersión entre las dos variables, y trazar una línea de tendencia, se puede apreciar que existe una aparente relación lineal inversa entre las variables, lo que a priori nos haría pensar que el coeficiente de correlación de Pearson debería ser negativo.

Diagrama de Dispersión

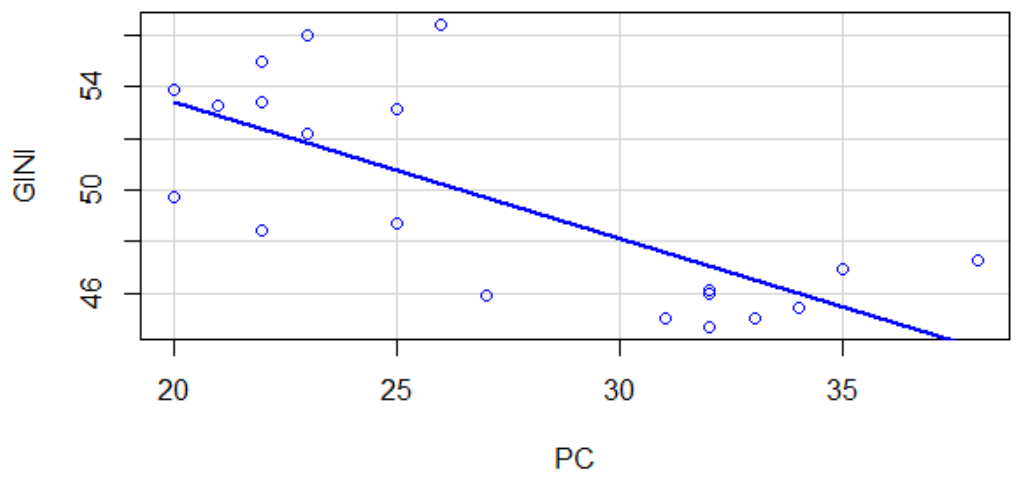

Figura 5. Diagrama de Dispersión

Fuente: Elaboración Propia.

Finalmente se calcula el coeficiente de correlación lineal de Pearson, lo cual arroja el siguiente resultado:

$$
r_{x y}=-0.7319136
$$

Esto indica que existe una relación lineal inversa entre las variables, Índice de Percepción de Corrupción y el Coeficiente de GINI, y dicha relación es bastante alta. Esto significa que, cuando una variable aumenta la otra disminuye, es decir, las variables se mueven en sentidos opuestos. Cuando el Índice de Percepción de Corrupción aumenta, el Coeficiente de GINI disminuye; sin embargo, este resultado no implica existencia de causalidad, es decir, esto no quiere decir que los cambios en el Índice de Percepción de Corrupción tengan un efecto sobre el Coeficiente de GINI, o viceversa, simplemente muestra que, para el período de análisis, existe una relación matemática inversa entre las dos variables. 


\section{Conclusiones.}

- La manufactura, el comercio y el sector petrolero son la base de la economía ecuatoriana, ya que estos son los 3 sectores dinamizadores; por un lado, la manufactura en promedio para el periodo de estudio representó el 11,87\%; el comercio con el 10,55\%; y el petróleo y minas 10,42\% en relación con el VAB; en tanto el sector de servicio doméstico y acuicultura tienen una baja representatividad para la economía.

- El índice de Percepción de Corrupción y el Producto Interno Bruto presentan una alta relación entre si, es decir a mayor IPC menor es el crecimiento económico, en tanto que a menor IPC mayor es el PIB, por lo que podemos inferir que el índice de percepción de corrupción a lo largo del periodo de estudio ha mejorado empezando con 26 puntos para el año 2000 en tanto para el 2019 con 38 puntos, sin lugar a duda es un tanto alentador observar como el IPC ha bajado dentro del periodo de estudio, claro está que aún falta mejorar este aspecto que mina el crecimiento económico y con ello el desarrollo económico del país.

- En cuanto al coeficiente de gini y el IPC tenemos una correlación negativa lo cual significa que a mayor desigualdad registrada por el coeficiente de gini, mayor fue el IPC registrado, acompañado además en estos años con una contracción del PIB por lo que se determina que, al existir IPC alto, la desigualdad registrada será también alta, y esto mina el crecimiento económico.

\section{Referencias bibliográficas.}

Astroulakis, N. (2013). Desafiando a la economía convencional: Un paradigma ético del desarrollo. Problemas Del Desarrollo, 44(175), 33-53. https://doi.org/10.1016/s0301-7036(13)71901-1

Astroulakis, N. (2019). The Applied-Ethical Structural Synthesis of International Development. Problemas Del Desarrollo. Revista Latinoamericana de Economía, 50(197), 87-110. https://doi.org/10.22201/iiec.20078951e.2019.197.65856

Bautista, O. (2012). El problema de la corrupción en América Latina y la incorporación de la ética para su solución. Espacios Públicos, 15(35), 48-62.

Beteta, H., \& Moreno-Brid, J. (2012). El desarrollo en las ideas de la CEPAL. Economíaunam, 9(27), 76-90.

Bielschowsky, R. (2006). Vigencia de los aportes de Celso Furtado al estructuralismo. Revista de La CEPAL, 2006(88), 7-15. https://doi.org/10.18356/3e1e77a8-es

Campos, J. E., \& Pradhan, S. (2009). Las múltiples caras de la corrupción. Aspectos vulnerables por sectores. 389.

CEPAL. (2016). Desarrollo inclusivo en América Latina.

Franco, H., \& Ramirez, A. (2005). El modelo Harrod-Domar: implicaciones teóricas y 
empíricas. Ecos de Economía, 9(21), 127-151.

Frank, A. (2005). Redalyc.TEORÍA DE LA DEPENDENCIA. LA DEPENDENCIA DE CELSO FURTADO.

Guerra, C., \& González, I. (2015). La relación dinámica del valor agregado bruto, la producción mercantil y el gasto material. Su importancia para la toma de decisiones. Acta Universitatis Agriculturae et Silviculturae Mendelianae Brunensis, 53(9), 1689-1699.

http://publications.lib.chalmers.se/records/fulltext/245180/245180.pdf\%0Ahttps://h dl.handle.net/20.500.12380/245180\%0Ahttp://dx.doi.org/10.1016/j.jsames.2011.03 .003\%0Ahttps://doi.org/10.1016/j.gr.2017.08.001\%0Ahttp://dx.doi.org/10.1016/j.p recamres.2014.12

Hernández, P. L., \& Herrera, P. (2018). Corrupción, Gobernabilidad y Crecimiento Económico. Un estudio empírico. Acta Universitaria, 28, 24-34. https://doi.org/10.15174/au.2018.2006

Hofman, A. (1994). No.21-1994AndreHofman.pdf. BCE. https://www.bce.fin.ec/cuestiones_economicas/images/PDFS/1994/No21/No.211994AndreHofman.pdf

Internacional, X. D. E. T. (2014). Indice de percepción de la corrupción 2014 enfoque Mexico.

Iturralde, C. A. (2019). Los paradigmas del desarrollo y su evolución: Del enfoque económico al multidisciplinario. Retos, 9(17), 7-23. https://doi.org/10.17163/ret.n17.2019.01

López, L., \& Sánchez, I. (2013). Crecimiento económico , corrupción e instituciones en México. Redalyc.

Marjanović, V. (2016). Structural Changes and Structural Transformation in a Modern Development Economy. Economic Themes, 53(1), 63-82. https://doi.org/10.1515/ethemes-2015-0005

Martínez-Echevarria, M., \& Crespo, R. (2011). Aristóteles y el pensamiento económico: una introducción. Revista Empresa y Humanismo, 14(2), 5-9.

Medina, F. (2001). Consideraciones sobre el índice de Gini para medir la concentración del ingreso.

Montalvo, J. O. (2017). Ecuador 1980-1990: Crisis, ajuste y cambio de régimen de desarrollo. America Latina En La Historia Economica, 24(1), 210-242. https://doi.org/10.18232/alhe.v24i1.724

Mundial, B. (2016). Macroeconómia. In Banco Mundial. https://www.bancomundial.org/es/topic/macroeconomics/overview

Núñez Velázquez, J. J. (2006). La desigualdad económica medida a través de las curvas de Lorenz. Revista de Metodos Cuantitativos Para La Economia y La Empresa, 2(1), 67-108.

ONU. (2005). Clasificación industrial internacional uniforme de todas las actividades 
económicas CIIU (Issue 4).

Ornelas, J. (2012). Volver al desarrollo. Problemas Del Desarrollo, 168(43), 7-35.

Ortiz, E. (2012). Edisonortiz.Pdf. TENDENCIAS Revista de La Facultad de Ciencias Económicas y Administrativas. Universidad de Nariño, XII XIII(1), 9-35.

Palacios, J. (2014). Efectos de la corrupción sobre el crecimiento económico. Un análisis empirico internacional Effects of corruption on economic growth-An international empirical analysis. 109-126.

Palestina, I. (2018). Corrupción y crecimiento económico: las percepciones de la sociedad colombiana. CES Derecho, 9(1), 59-72. https://doi.org/10.21615/cesder.9.1.4

Petit, J. (2014). LA TEORIA ECONONOMICA DE LA INTEGRACION Y SUS PRINCIPIOS FUNDAMENTALES. Principios De La Investigación, 15(4), 36. http://saber.ucv.ve/ojs/index.php/rev_ac/article/view/7905

Restrepo, 2015. (2015). UNA REVISIÓN ANALÍTICA SOBRE EL PAPEL DE LA TIERRA EN LA TEORÍA ECONÓMICA DE DAVID RICARDO*. Redalyc, XXIII(1), 103-114.

Restrepo, L., \& González, J. (2007). De Pearson a Spearman. Rev Col Cienc Pec, 20, $183-192$.

Reyes, G. (2001). Teoría de la Globalización: Bases Fundamentales. Nómadas: Critical Journal of Social and Juridical Sciences, II(3), 8. https://doi.org/10.5209/NOMA.37915

Roark, G., Urrutia, S., Jaurenguiberry, M., Diana, P., \& Daiana, O. (2013). Actividades predominantes a nivel Nacional, Regional y Local de las Pymes Industriales Argentinas. Ciencias Administrativas, 1, 4.

Rodero, A. (2012). "Introducción a la economía." In Revista de Fomento Social. https://doi.org/10.32418/rfs.2012.265.1827

Schmidtke, T., Koch, H., \& García, V. C. (2014). Los sectores económicos en América Latina y su participación en los perfiles exportadores Los sectores económicos en América Latina y su participación en los perfiles exportadores. http://library.fes.de/pdf-files/bueros/mexiko/14815.pdf

Sen Amartya. (2009). Desarrollo económico y libertad.

Valdez, J. (2016). Crecimiento económico y desarrollo. Un dilema de la realiadad actual. Flacso Ecuador, XI, 16. https://repositorio.flacsoandes.edu.ec/bitstream/10469/11013/1/REXTN-MS39-07Valdes.pdf

Vélez T., J. M. (2018). La Ley Petty-Clark en el Área Metropolitana del Valle de Aburrá en Colombia, en el periodo 2000-2016. Análisis Económico, 33(82), 95-110. https://doi.org/10.24275/uam/azc/dcsh/ae/2018v33n82/velez

Vergara, C. A., \& Ortiz, D. (2016). Desarrollo sostenible: enfoques desde las ciencias económicas $* /$ Sustainable development: approaches from 
economics/Desenvolvimento sustentável: abordagens da ciência econômica. Apuntes Del CENES - Redalyc, 35(62), 15-52. https://doi.org/10.19053/22565779.4240

Wielandt, G., \& Artigas, C. (2007). La corrupción y la impunidad en el marco del desarrollo en América Latina y el Caribe: un enfoque centrado en derechos desde la perspectiva de las Naciones Unidas. CEPAL División de Desarrollo Social, 52.

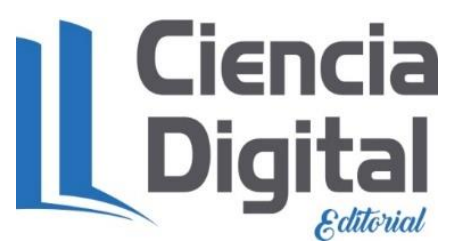




\section{PARA CITAR EL ARTÍCULO INDEXADO.}

Nievecela Lema, J. M., Covri Rivera, D., \& Castillo Ortega, Y. (2021). El Valor Agregado Bruto, la corrupción y la desigualdad en Ecuador. Un análisis descriptivo y $\begin{array}{llll}\text { correlacional. } & \text { Visionario } & \text { Digital, } & \text { 30-48, }\end{array}$ https://doi.org/10.33262/visionariodigital.v5i2.1634

\section{Ciencia Ligital}

El artículo que se publica es de exclusiva responsabilidad de los autores y no necesariamente reflejan el pensamiento de la Revista Visionario Digital.

El artículo queda en propiedad de la revista y, por tanto, su publicación parcial y/o total en otro medio tiene que ser autorizado por el director de la Revista Visionario Digital.
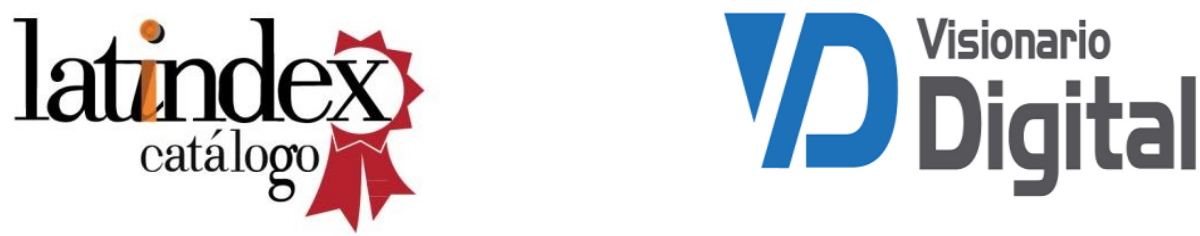